\title{
Reflexionando sobre las relaciones entre política social y estructura social ${ }^{1}$
}

\author{
José Adelantado \\ José Antonio N oguera \\ Universitat Autònoma de Barcelona. D epartament de Sociologia \\ Seminari d'Anàlisi de Polítiques Socials (SAP.S) \\ 08193 Bellaterra (Barcelona). Spain
}

Resumen

Este trabajo presenta los principal es debates sostenidos en el SAP.S (Seminari d'A nàlisi de Polítiques Socials) de la U AB, con el objeto de elaborar un marco teórico que permita analizar las relaciones entre política social y estructura social. Partiendo de la crítica a los modelos de Esping-Andersen, de los neomarxistas como 0 ffe 0 Gough, o de teóricos social es como $\mathrm{H}$ abermas, llegamos a una idea de la estructura social como compuesta por esferas de acción, desigualdades y actores colectivos. Por su parte, la política social se concibe a la vez como compensadora y constituidora de las desigualdades, y su relación con la estructura social es de carácter reflexivo.

Palabras clave: política social, estructura social, desigualdades, Estado de bienestar.

\section{Abstract}

This article presents the main debates that have developed in the SAP.S (Social Policy Analysis Seminar, $U A B$ ), in order to build a theoretical framework which should be able to analyse the relations between social policy and social structure. We start from some criticisms to the ideas of scholars like Esping-Andersen, neo-marxist authors like $\mathrm{O}$ ffe or Gough, and social theorists like $\mathrm{H}$ abermas, and we arrive to the defence of three ideas: social structure is composed of some spheres of activity, some inequalities, and some collective social actors; social policy acts both compensating and constituting social inequalities; and the relation between social policy and social structure has a reflexive nature.

Key words: social policy, social structure, inequalities, welfare state.

I. Texto sin modificar de una intervención en las Primeres Jornades de Recerca del $D$ epartament de Sociologia de la UAB (17 y 18 de febrero de 1999). El texto completo y la bibliografía en que se basa pueden encontrarse en el Working Paper $n^{\circ}$ I del SAP.S (D epartament de Sociologia, enero de 1998) y, en diversas versiones, ha sido parcialmente publicado en la Revista Internacional de Sociología n 22 (enero-abril de 1999) y en la Revista M exicana de Sociología nº 3 de 1998. 
La presente intervención se propone únicamente exponer algunos de los deba tes teóricos que hemos desarrollado en el seno del Seminari d'Anàlisi de Polítiques Socials (SAP.S) desde hace unos dos años, con el objeto de plantear un estudio sistemático de las relaciones entre política social y estructura social, y de aplicarlo al caso del Estado español. A continuación se pasa revista a lo que podríamos Ilamar la «historia interna» de esos debates: las diferentes perspectivas conceptuales que hemos ido discutiendo y adoptando, y los problemas que nos han suscitado tanto teóricamente como en su posible aplicación empírica; concluiremos mencionando al gunas de las primeras aplicaciones empíricas de esta perspectiva, aún en curso, que estamos iniciando en estos momentos.

La intención de analizar la política social (o los «Estados de bienestar») en referencia a la estructura social (o a la «estratificación social») debe relacionarse, sin duda, con la aparición del famoso libro de G. Esping-A ndersen, Lostres mundos del Estado de bienestar, en 1990 y los debates que suscitó. Lo que percibíamos era la necesidad de partir de una crítica a los planteamientos de la citada obra, aunque asumiéndolos parcialmente. La crítica a Esping-Andersen, y la relectura de otras aportaciones teóricas sobre la política social (como las de Polanyi, O ffe o G ough), fue el inicio de un replanteamiento que, a grandes rasgos, cabría esquematizar como sigue.

En una primera fase, contrapusimos las aportaciones de estos autores a las de Esping-Andersen. Ciertamente al gunos aspectos de la obra del segundo resultaban provechosos:

- En primer lugar, la idea de entender los «regímenes de bienestar» como algo más amplio que un mero conjunto de políticas públicas, por cuanto éstas se hallan conectadas con sistemas determinados de estratificación social y son el resultado de alianzas y dinámicas de conflicto muy diversas entre los actores sociales.

- Por otro lado, el concepto de desmercantilización funciona en EspingAndersen como indicador que permite la comparación entre diferentes regímenes de bienestar y su conexión con la estructura social, al tiempo que ofrece un claro referente de connotaciones normativas.

Sin embargo, algunas deficiencias de este planteamiento se ponen de manifiesto al trabajar las aportaciones de Polanyi, O ffe o G ough, quienes, con intenciones y métodos distintos, ya habían iniciado bastante antes que Esping-Andersen la tarea de relacionar políticas social es con estructura social:

- En primer lugar, la obra de Esping-Andersen, a diferencia de la de los autores citados, ha dado lugar entre sociólogos y politólogos a un cierto «fetichismo taxonómico» preocupado en exceso por la clasificación de los diferentes países bajo etiquetas de diferentes tipos de «Estados» 0 «regímenes de bienestar». Este enfoque, que puede ser muy útil para los estudios comparativos y puramente descriptivos, sin embargo tiene poco poder explicativo: lo importante, desde estos otros autores, no es bajo qué eti- 
queta clasificar un determinado país o una determinada política, sino lo que las políticas sociales concretas hacen o dejan de hacer. $\mathrm{N}$ o se puede fetichizar la idea de «el» Estado de bienestar, o de «los diferentes tipos» de Estados de bienestar, sino que conviene analizar las pol íticas sociales diversas y concretas en cada caso.

- En segundo lugar, el énfasis de Esping-Andersen en la desmercantilización introducía un sesgo político «socialdemócrata» según el cual el Estado del bienestar - sobre todo el de tipo «universalista», hacia el que se inclinaban entonces las simpatías del autor- «compensa» y hace soportables las desigual dades surgidas del mercado capitalista; este énfasis «desmercantilizante» oscurecía, asimismo, una parte importante del asunto a tratar: impedía ver empírica e históricamente cómo las políticas sociales han ido y van también ligadas a importantes procesos de mercantilización en las sociedades capitalistas, e incluso pueden constituirlos, afianzarlos y reproducirlos. En el análisis de las relaciones entre política y estructura social, el concepto de mercantilización es previo al de desmercantilización, o, como mínimo, debe yuxtaponerse al mismo; las políticas sociales pueden tener - y tienen - efectos mercantilizadores tanto como desmercantilizadores, en un sentido más global de lo que Esping-Andersen supone; por ejemplo: la introducción de una política de seguros de desempleo «desmercantiliza» para Esping-Andersen, en el sentido de que permite sobre vivir determinados períodos de tiempo al margen de la venta de la fuerza de trabajo en el mercado; pero, sin embargo, «mercantiliza», en el sentido de que el cobro de las prestaciones va necesariamente ligado a la participación en el mercado de trabajo: en muchos casos, es una condición de posibilidad para el establecimiento pleno de éste último; y en la mayoría reproduce la «proletarización activa» (en expresión de 0 ffe) de la fuerza de trabajo, e introduce una nu eva desigual dad no existente hasta entonces entre desempleados con o sin derecho a la prestación.

Por tanto, en esta primera fase, y al amparo de la relectura de Polanyi, O ffe o Gough, consideramos conveniente pasar:

- del análisis de «modelos» de Estados de bienestar al análisis de políticas sociales concretas; y

- de una concepción «compensatoria» de la política social a una al mismo tiempo «constitutiva» de las desigualdades y de los procesos de mercantili zación en las sociedades capitalistas occidentales.

Cabría añadir también que, frente a la utilización en el libro de EspingAndersen del concepto de «estratificación social», nos pareció más oportuno optar por el de «estructura social»; el primero tiene connotaciones funcionalistas, presupone que los grupos sociales se configuran como «estratos», y que su clasificación teórica como tales responde a criterios arbitrarios del teórico o investigador, no a divisorias o contradicciones reales, existentes en la propia sociedad; 
el segundo concepto nos parecía, por tanto, más apto para hablar de desigual dades.

En un segundo momento surgieron a la luz las dificultades que, a su vez, tenían los enfoques neomarxistas de autores como 0 ffe o G ough, quienes habían sido de utilidad para la crítica del esquema de Esping-Andersen; estas limitaciones pueden resumirse en tres puntos:

- En primer lugar, la estructura social se planteaba únicamente en términos de estructura de dases, lo que daba lugar a dos problemas: excluía del mode lo a otras desigualdades relevantes a la hora de estudiar la política social, como las de género, edad, etc., y nos introducía en la interminable discusión sobre la definición y operativización de la «clase social». A este respecto, los model os de estructura de clases de Wright y de Roemer parecían ofrecer una solución provisional a tales problemas: al plantear las clases en conexión con la explotación de cualquier tipo de recursos, podían incluir la explotación de fuerza de trabajo por los varones en el caso del ámbito doméstico, a la vez que dar definiciones y operativizaciones precisas de las clases y las desigualdades. Sin embargo, las limitaciones del enfoque de Roemer fueron ya puestas de manifiesto por el propio Roemer y por W right. $\mathrm{N}$ o entraremos aquí en los detalles de sus model os de clases ni en sus dificultades teóricas y empíricas baste decir que el criterio de explotación tampoco parecía suficiente para dar cuenta de las numerosas divisorias que existen en las sociedades eapitalistas occidental es contemporáneas; además, la pretensión de estableeer un modelo de estructura de clases nos alejaba considerablemente de nuestro objeto prioritario de estudio, que era la política social: una postura más flexible y abierta al caso concreto parecía más conveniente en este caso. Por ejemplo, no era tan importante estudiar la explotación en base a la propiedad o no sobre medios de producción, cuando la política social nunca influye ni de lejos en ésta última; o podíamos hablar de impactos de la política social sobre la distribución de la renta, la distribución de la riqueza, las desigualdades de posición en el mercado de trabajo, las divisorias internas dentro de los colectivos de trabajadores y trabajadoras, asalariados y asalariadas, etc., sin necesidad de plantearnos a cada paso qué es y qué no es lo que constituye una «clase».

- En segundo lugar, los modelos neomarxistas resultaban excesivamente «mercadocéntricos»: tendían a analizar únicamente las dinámicas de mercantilización-desmercantilización inducidas por la política social. Pero esto dejaba fuera otras posibles dinámicas social es ligadas también a la misma: era necesario introducir también la cuestión de cómo la política social influía en los ámbitos político (el propio Estado) y doméstico (el hogar familiar). En un primer momento, nos planteábamos procesos tales como los de «burocratización» como paralel os a los de «mercantilización» (siguiendo a Weber y a $\mathrm{H}$ abermas), y dejábamos el problema del ámbito doméstico como un problema más de explotación según el modelo roe 
meriano. Pero ninguno de los intentos por encajar todo el esquema pare cía satisfactorio.

- Relacionado con este último problema estaba también el molesto aire «funcionalista» que algunos de los enfoques neomarxistas asumían implícitamente: la política social se estudiaba más por sus «funciones» que por sus «causas» y sus efectos concretos sobre las desigual dades concretas; «funciones» que, en el caso de G ough - e implícitamente también de O ffe- , quedaban clasificadas triádicamente como de acumulación, reproducción y legitimación, las cuales, a su vez, parecían conectarse conceptualmente con los ámbitos del mercado, la sociedad y el Estado. Tras varios intentos de extraer conclusiones o hi pótesis de este esquema, aparecieron otros problemas además de su funcionalismo: no era empíricamente muy informativo; cualquier cosa podía clasificarse como acumuladora, reproductora y legitimadora, y al mismo tiempo era difícil establecer las fronteras empíricas entre tales «funciones», dónde acababa una y empezaba la otra; por otro lado, al menos las funciones de «acumulación» y «legitimación», y en parte también la de «reproducción», nos conducían a un estudio ciclópeo a gran escala de la sociedad como un todo, que estaba lejos de nuestras posibilidades y de nuestras intenciones (las relaciones entre política social y estructura social eran sólo una parte del esquema teórico de G ough). Por estos motivos abandonamos también estos conceptos como poco relevantes para nuestra tarea.

Por tales razones, en una tercera fase adoptamos un esquema conceptual al go distinto, esta vez basánd onos fundamentalmente en la teoría de las sociedades capitalistas tardías desarrollada por H abermas en su Teoría de la acción comunicativa en 1981 (y adoptada por O ffe en sus trabajos de los años ochenta sobre el Estado de bienestar). Pasamos a hablar de esferas de la estructura social, concretamente de cuatro: la mercantil, la estatal, la doméstico-familiar y la relacional (ésta última inspirada en lo que $\mathrm{H}$ abermas Ilama «esfera pública» - 0 en la tradición gramsciana de análisis de la «sociedad civil»-, pero completada con las aportaciones de otros autores como M ingione sobre la esfe ra de los asuntos asociativos y comunitarios). La idea de las esferas como contextos de acción estructurados (parecida a la idea de «regiones» en Giddens o de «campos» en Bourdieu) resultaba adecuada para definir diferentes ámbitos de la estructura social, en tanto en cuanto fuesen más allá de la mera situación de los individuos en base a desigualdades concretas, para constituir el contexto dado con el que se encuentran. Para decirlo con un ejemplo, una cosa es ocupar el lugar de ama de casa en el ámbito doméstico-familiar, y otra previa ana líticamente el haber nacido en una sociedad con una determinada estructuración de esa esfera (más o menos patriarcal, con un tipo u otro de familia, etc.).

La idea de las esferas solucionaba el problema del «mercadocentrismo» neomarxista en cuanto a los impactos de la política social, permitía abrir el abanico de desigualdades objeto de la misma más allá de una mera «estructura de 
clases», y además abandonaba la tríada de «funciones» de Gough como irrele vante. Sin embargo, el problema que nos planteamos a continuación fue el de la «conexión» de las esferas entre sí, en función de las desigualdades; en principio, siguiendo el esquema habermasiano, la conexión parecía darse en términos de «roles» sociales; a los que $\mathrm{H}$ abermas enumera, que nos parecían insuficientes, añadimos algunos, en parte basados aún en la teoría de Roemer (como los de explotador y explotado, etc.).

Sin embargo, en una cuarta fase el esquema anterior fue a la vez modificado, ampliado y flexibilizado. Podemos enumerar los diferentes pasos que dimos hasta la configuración de nuestro enfoque actual (sin duda aún abierto y nunca definitivo):

- La estructura social, en un sentido amplio, queda configurada en base a tres dimensiones de análisis: a las esferas - ya citadas - se añaden los ejes de desigualdad - que sustituyen a los «roles» de H abermas- y los actores colectivos - que dan cuenta del aspecto activo, de estructuración de la propia estructura (Giddens), y de la formación de las políticas sociales.

- Las cuatro esferas quedan ligadas con la política social mediante lo que llamamos procedimientos. las políticas sociales pueden tener impactos diversos de acuerdo con la distribución de provisión de bienestar social que favorezcan entre las distintas esferas: aś, pueden mercantilizar o desmercantilizar, estatal izar o desestatalizar, familiarizar o desfamiliarizar, y comunitarizar 0 descomunitarizar.

- Los ejes de desigualdad constituyen una lista en principio abierta, en la que sin duda entran las desigual dades de clase (relacionadas con el merca do de trabajo y la distribución de la riqueza y los recursos) y de género, pero también otras ya existentes (de edad, étnicas, etc.) o que pueden ser propiciadas por la propia política social (desigualdades en cuanto a los derechos sociales, de ciudadanía, etc.), retomando así el argumento de los efectos «constituidores» de desigualdad que ésta puede tener. Además, empíricamente se trataría de ver si una política social concreta (o una medida de política social) arroja un saldo igualizador, reproductor o polarizador en torno a determinados ejes de desigualdad.

- No existiría una conexión automática, por decreto teórico, entre las diversas esferas, sino conexiones históricas contingentes y cambiantes, como contextos de actividad históricos que son, y distinguibles más en el plano analítico que en la actividad cotidiana de los individuos. Asimismo, la cuestión de la conexión entre las esferas, las desigualdades y los actores no puede establecerse de forma automática o mecánica: las desigual dades de clase 0 de género (y otras) son transversales a las esferas, se manifiestan y tienen efectos palpables en distintos contextos de actividad, aunque podamos intuir que su constitución histórica está más o menos ligada a una esfera particular (pero este sería un tema alejado de nuestro objeto de estudio).

- Por último, si queremos realmente huir de cualquier positivismo - como es nuestro caso- y considerar la realidad social como histórica y contra- 
dictoria, no podemos otorgar respectivamente a la estructura social y a la política social los papeles de «variable explicativa» y «variable explicada» 0 viceversa. $\mathrm{H}$ emos de ser conscientes de que estamos aislando a efectos analíticos al gunos aspectos concretos de cada una de ellas (tal y como hacía Weber en La ética protestante...) con vistas a establecer relaciones entre las mismas en un doble sentido: la estructura social condiciona y da forma a las políticas sociales, al tiempo que éstas influyen y modifican la propia estructura social de la cual nacen; siguiendo a Giddens, la política social puede entenderse así como una instancia a la vez condicionada estructuralmente y estructuradora; 0 , dicho de otro modo, como un fenómeno más de reflexividad en las sociedades contemporáneas: si se permite el antropomorfismo, mediante la política social - entre otras cosas- la estructura social «actúa sobre sí misma» y se modifica y reestructura. La relación entre ambas, por tanto, es reflexiva y circular.

A efectos empíricos, esto tiene consecuencias claras: un análisis «históricosociológico» del caso concreto es necesario para estudiar tanto la formación (a partir de la estructura social) como el impacto (sobre esa misma estructura social) de cualquier medida de política social. Será necesario analizar, en el primer caso, la dinámica interna de las esferas que influyan en la política concreta, los ejes de desi gualdad sobre los que pretende actuar y que la originan, o las presiones de actores concretos sobre la misma; y en el segundo caso, los procedimientos que implementa, el efecto sobre las desigualdades o la constitución de las mismas, así como la posible respuesta social por parte de actores colectivos. Y ésta es la tarea que en estos momentos estamos iniciando, respecto de al gunas políticas concretas a un triple nivel (estatal, autonómico - C atalunya y País Vasco - y local), con una ayuda de la D GICYT concedida a tal efecto, que nos permitirá realizar trabajo de campo que vaya más allá del habitual estudio en términos estadísticos y documentales.

\section{Bibliografía}

EsPIN g An DERSEN, Gosta (1990). Los tres mundos del Estado del bienestar. Valencia: Edicions Alfons el M agnànim, 1993.

Gou G, Ian (1979). Economía política del Estado del bienestar. M adrid: Blume, 1982. H ABERM AS, Jürgen (1981). The Constitution of Society. Berkeley: University of California Press.

O fFe, Claus (1983). Contradiccionesen el Estado del bienestar. M adrid: Alianza, 1990. POLAN YI, Karl (1944). La gran transformación. M adrid: La Piqueta, 1990.

RoEm ER, John (1982). Teoría general de la explotación y de las clases. M adrid: Siglo XXI, 1989.

W RIGHT, Erik O lin (1989). «Reflexionando, una vez más, sobre el concepto de estructura de clases», a Julio Carabañ A; Andrés de Fran Cisco (comps.) (1994). Teo rías contemporáneas de las clases sociales. M adrid: Editorial Pablo I glesias. 